\title{
The Research of the Interactive Relationship Based on the VAR Model between the Prices of Pork, Soybeans and Corn
}

\author{
Siyu Mo, Wen Han \\ ${ }^{1,2}$ Financial College, Guizhou College of Finance and Economics, Guizhou 550025, China

\section{基于 VAR 模型的猪肉价格、大豆价格与 玉米价格互动关系研究}

默思羽, 韩 雯

贵州财经大学金融学院, 贵州 550025, 中国

\begin{abstract}
The research of the interactive relationship based on the VAR model between the prices of pork, soybeans and corn in Guizhou, provides reasonable reference to make effective policy of adjusting pork price regularly, and establish the necessary warning system for the production of the pigs. The article take the example of the prices of pork, soybeans and corn from August 1th, 2014 to April 30th, 2015 in Guizhou uses the Vector Auto regression model, and through the impulse response function and the variance decomposition and other methods to systematically analyzes the mutual influence between the prices. The results shows that the pork price is influenced most by itself, but it is influenced relatively less by the prices of corn and soybean. The prices between corn and soybean have interactive effect but the degree is small. Finally, we propose method to maintain the stability of the pork market price in Guizhou.
\end{abstract}

Key words: VAR model; pork price; soybean price; corn price; interactive relationship; Guizhou province

\section{摘要}

基于 VAR 模型在贵州省猪肉价格、大豆价格与玉米 价格之间的互动关系研究, 以期为制定有效的猪肉价
格调控政策、建立必要的生猪生产预警机制提供合理 的决策参考依据。文章以 2014 年 8 月 1 日-2015 年 4 月 30 日贵州省猪肉、大豆、玉米三类日价格数据为 例, 运用向量自回归 (VAR) 模型, 通过脉冲响应 函数与方差分解等方法, 系统分析了其价格间的相互 影响程度。结果表明, 猪肉价格受其自身的影响最大, 但其对玉米价格和大豆价格的影响相对较小, 玉米价 格和大豆价格之间有一定的互相影响作用, 但影响程 度均较小。最后, 提出维护贵州省猪肉市场价格的稳 定的方法。

关键词: VAR 模型；猪肉价格；大豆价格； 玉米价格; 互动关系; 贵州省

\section{1.引言}

猪肉作为贵州省畜牧业产量占比最高的产品, 年生猪 出栏数 1800 余万头, 猪肉年总产量达到 165 万吨以上。 作为贵州省城乡居民日常消费的主要肉类食品之一 [1], 其价格的波动不仅直接影响居民对猪肉产品的 需求, 还影响猪肉市场秩序与农村经济的稳定。2014 年8月-2016年4月期间贵州省猪肉价格浮动比率为 $25 \%$, 大豆价格浮动比率为 $100 \%$, 玉米价格浮动比 率为 $150 \%$ 。 ${ }^{1}$ 近年来, 贵州省在积极推进生猪规模化 养殖, 主要采取散养、小规模、中规模及大规模的养 殖模式。不同规模的生猪养殖模式对配合饲料和饲料 \footnotetext{
而得。

1数据来源: 贵州省农经网.价格信息 http://www.gznw.gov.cn 整理
} 


\section{Risk Analysis and Crisis Response in Big Data Era (RAC-16)}

粮的需求均有不同幅度的增加, 这是导致了近年来贵 州生猪养殖成本持续上涨的主要原因。无论是传统散 养还是规模化养殖, 均选择浓缩饲料搭配玉米和大豆 等配合饲料对猪进行喂养[2]。在生猪养殖过程中, 玉米、大豆等配合饲料费用约占养殖总成本的 $50 \%$, 特别是大规模养殖生猪耗粮数量增加 $43 \%$, 每 $50 \mathrm{~kg}$ 主 产品多消耗粮食 $32.9 \mathrm{~kg}$ 。贵州作为粮食产销平衡的省 份, 适度扩大生猪养殖规模的发展潜力受到一定的限 制, 尤其是粮食物流瓶颈及成本制约着其他地区玉米 输入贵州。因此配合饲料费用的增加直接导致生猪养 殖成本上涨, 从而对猪肉价格有着直接的影响[3]。

目前, 国内对影响猪肉价格的研究主要包括几个 方面:（1）生猪价格波动原因研究。站立强[4]运用 时间序列法分析得出: 能繁母猪数量与生猪出栏量以 及生猪价格之间的循环因果关系是导致生猪价格周 期波动的根本原因。宁做凉, 乔娟[5]等对2000年1月 -2009年10月间生猪价格波动的特征、影响及导致生 猪价格波动的各种原因进行分析得出投入品价格、居 民收入、替代品价格、互补品价格及其他因素共同影 响猪肉价格波动。张立中, 刘倩倩[6]等, 采用VAR 模型, 对我国生猪价格波动的影响因素进行了分析, 结果发现生猪生产成本对生猪价格的冲击最大。(2) 生猪价格波动规律研究。潘方卉, 李翠霞[7]等应用 (MSIH(3)-AR(1))模型, 对我国生猪价格的非线性波 动规律进行了实证分析得出生猪价格具有不同的波 动水平、转移概率和持续期。李威夷[8]针对生猪价 格的波动, 运用 GARCH族模型和先进的B-N数据分 解技术对生猪价格波动进行实证分析表面生猪价格 的波动具有集聚性, 外部冲击幅度越大, 价格的波动 就越剧烈。张晓东, 王超群[9]等运用VAR模型, 分 析得出生猪价格波动与猪肉价格波动、玉米价格波动、 仔猪价格波动是一个动态的系统。(3) 生猪价格预警 研究。康艺之, 郑业鲁, 林伟君, 方伟[10]等采用黑 色预警方法, 以价格波动率为警情指标, 建立了我国 猪市价格异常波动预警模型并展开预警。赵瑞莹, 陈 会英, 杨学成[11]等基于 $\mathrm{BP}$ 人工神经网络建立了生猪 价格风险预警模型。然而, 目前还没有学者针贵州省 猪肉价格与大豆价格、玉米价格三者之间的互动关系 进行实证研究。只有了解掌握了贵州省猪肉价格波动 与大豆、玉米价格之间的互动关系, 才能更有针对性 的对贵州省生猪市场进行管理和调控, 保障贵州省生 猪产业健康发展。

因此, 本文选取大豆价格和玉米价格作为影响猪 肉价格的参数, 借助向量自回归模型, 运用脉冲响应 函数和方差分解方法分析了大豆、玉米与猪肉三者价 格之间的互动关系, 以期为地方政府有效的调控猪肉
市场的供求关系、农户采取合理的生产决策以及猪肉 市场交易商掌握较准确的交易信息提供参考。

\section{2.理论阐述}

\subsection{VAR 模型}

向量自回归 (Vector Auto regression, VAR) 模型是 基于数据的统计性质建立模型, VAR 模型把系统中 每一个内生变量作为系统中所有内生变量的滞后值 的函数来构造模型, 从而将单变量自回归模型推广到 由多元时间序列变量组成的“向量”自回归模型。VAR 模型在经济学研究中通常用于相关时间序列系统的 预测和随机扰动对变量系统的动态冲击, 从而解释各 种经济冲击对经济变量形成的影响。

$\operatorname{VAR}(p)$ 模型的数学表达式是:

$$
\mathrm{y}_{\mathrm{t}}=\mathrm{A}_{1} \mathrm{y}_{\mathrm{t}-1}+\cdots \cdots+\mathrm{A}_{\mathrm{P}} \mathrm{y}_{\mathrm{t}-\mathrm{p}}+\mathrm{BX}_{\mathrm{t}}+\varepsilon_{\mathrm{t}}
$$

其中: $y t$ 是 $\mathrm{k}$ 维内生变量向量, $\mathrm{Xt}$ 是 $\mathrm{d}$ 维外生变量 向量, $p$ 是滞后阶数, 样本个数为 $T \circ k \times k$ 维矩阵 $A_{1}, \ldots$, $\mathrm{Ap}$ 和 $\mathrm{k} \times \mathrm{d}$ 维矩阵 $\mathrm{B}$ 是要被估计的系数矩阵。 $\mathrm{et}$ 是 $\mathrm{k}$ 维扰动向量, 它们相互之间可以同期相关, 但不与自 己的滞后值相关及不与等式右边的变量相关。

\section{2 脉冲响应函数}

脉冲响应函数 (IRF) 反映的是 VAR 模型中的一个内 生变量的冲击对其他内生变量所带来的影响。对于任 何一个 VAR 模型都可以表示成为一个无限阶的向量 MA $(\infty)$ 过程, 为:

$$
\begin{aligned}
\mathrm{Y}_{\mathrm{t}+\mathrm{q}} & =\mathrm{u}_{\mathrm{t}+\mathrm{q}}+\mathrm{C}_{1} \mathrm{u}_{\mathrm{t}+\mathrm{q}-1}+\mathrm{C}_{2} \mathrm{u}_{\mathrm{t}+\mathrm{q}-2}+\cdots+\mathrm{C}_{\mathrm{q}} \mathrm{u}_{\mathrm{t}}+\cdots \\
\mathrm{C}_{\mathrm{q}} & =\partial \mathrm{Y}_{\mathrm{t}+\mathrm{q}} / \partial \mathrm{u}_{\mathrm{t}}
\end{aligned}
$$

其中, $C_{Q}$ 中第 $\mathrm{i}$ 行 $\mathrm{j}$ 列元素表示的是, 当其他误差项 在任何时期都不变的条件下, 当第 $\mathrm{j}$ 个变量 $Y_{j t}$ 对应的 误差项 $\mathrm{u}_{\mathrm{jt}}$ 在 $\mathrm{t}$ 受到一个单位的冲击后, 对第 $\mathrm{i}$ 个内生 变量 $Y_{j t}$ 在 $t+s$ 期造成的影响。 $C_{q}$ 中第 $i$ 行 $j$ 列元素看 作是滞后期 $\mathrm{q}$ 的函数 $\partial \mathrm{Y}_{\mathrm{t}+\mathrm{q}} / \partial \mathrm{u}_{\mathrm{t}}, \mathrm{q}=1,2,3, \ldots$ 。它描 述了在 $\mathrm{t}$ 时期其他变量和早期变量不变的情况下, $\mathrm{Y}_{\mathrm{j}, \mathrm{t}+\mathrm{q}}$ 对 $\mathrm{u}_{\mathrm{j}, \mathrm{t}}$ 的一个冲击的反应过程, 称作脉冲响应函 数。

\section{3 方差分解}

方差分解(variance decomposition)是在于分析当系统 的一个变量受到结构冲击后, 以变量预测误差百分比 的形式反映变量之间的交互程度。其基本思路为:

$$
\mathrm{y}_{\mathrm{it}}=\sum_{\mathrm{j}=1}^{\mathrm{k}}\left(\mathrm{c}_{\mathrm{ij}}^{(0)} \varepsilon_{\mathrm{jt}}+\mathrm{c}_{\mathrm{ij}}^{(1)} \varepsilon_{\mathrm{jt}-1}+\mathrm{c}_{\mathrm{ij}}^{(2)} \varepsilon_{\mathrm{jt}-2}+\cdots\right),
$$


Risk Analysis and Crisis Response in Big Data Era (RAC-16)

$$
\mathrm{i}=1,2, \cdots, \mathrm{k}
$$

各括号中的内容是第 $\mathrm{j}$ 个扰动项 $\varepsilon_{j}$ 从无限过去到 现在时点对 $\mathrm{y}_{\mathrm{i}}$ 的影响, 求其方差, 假定 $\varepsilon_{\mathrm{j}}$ 无序列相关, 则有:

$$
\mathrm{E}\left[\left(\mathrm{c}_{\mathrm{ij}}^{(0)} \varepsilon_{\mathrm{jt}}+\mathrm{c}_{\mathrm{ij}}^{(1)} \varepsilon_{\mathrm{jt}-1}+\cdots\right)^{2}\right]=\sum_{\mathrm{q}=0}^{\infty}\left(\mathrm{c}_{\mathrm{ij}}^{(\mathrm{q})}\right)^{2} \sigma_{\mathrm{jj}},
$$$$
i, j=1,2, \cdots, k
$$

序列中可能存在的异方差, 采取对全部数据取自然对 数处理, 处理后的变量相应为 LNPP、LNSP 和 LNCP。

\section{4.模型构建与检验}

\section{1 单位根检验}

在进行实证分析前, 先采用 $\mathrm{ADF}$ 的检验方法对三个 变量检验。由表 1 可知, 在 $5 \%$ 显著水平下, 大豆价 格序列与玉米价格序列的 ADF 统计值均未超过临界

表 1. 单位根 $(A D F)$ 检验结果

\begin{tabular}{llllll}
\hline \multicolumn{1}{c}{ 序列 } & $\mathrm{ADF}$ 统计值 & $5 \%$ 临界值 & $1 \%$ 临界值 & 伴随概率 $\mathrm{P}$ & 结论 \\
\hline 猪肉价格 (LNPP) & -2.66 & -2.86 & -3.44 & 0.0816 & 不平稳 \\
大豆价格 (LNSP) & -4.20 & -2.86 & -3.44 & 0.0007 & 平稳 \\
玉米价格 (LNCP) & -4.34 & -2.86 & -3.44 & 0.0004 & 平稳 \\
$\Delta$ 猪肉价格 $(\Delta$ LNPP) & -27.64 & -2.86 & -3.44 & 0.0000 & 平稳 \\
\hline
\end{tabular}

注: $\triangle$ LNPP 表示 LNPP 的一阶差分

就是把第 $\mathrm{j}$ 个扰动项对第 $\mathrm{i}$ 个变量从无限过去到现在 时点的影响, 用方差加以评价的结果。此处还假定扰 动项向量的协方差矩阵 $\sum$ 是对角矩阵, 则 $\mathrm{y}_{\mathrm{i}}$ 的方差是 上述方差的 $\mathrm{k}$ 项的简单和, 为:

$$
\begin{array}{r}
\operatorname{Var}\left(\mathrm{y}_{\mathrm{it}}\right)=\sum_{\mathrm{j}=1}^{\mathrm{k}}\left\{\sum_{\mathrm{q}=0}^{\infty}\left(\mathrm{c}_{\mathrm{jj}}^{(\mathrm{q})}\right)^{2} \sigma_{\mathrm{jj}}\right\}, \\
\mathrm{i}=1,2, \cdots, \mathrm{k} \mathrm{t}=1,2, \cdots, \mathrm{T}
\end{array}
$$

$\mathrm{y}_{\mathrm{i}}$ 的方差可以分解成 $\mathrm{k}$ 种不相关的影响, 因此为了测 定各个扰动项相对 $\mathrm{y}_{\mathrm{i}}$ 的方差有多大程度的贡献, 定义 了如下尺度:

$$
\operatorname{RVC}_{j-i}(\infty)=\frac{\sum_{q=o}^{\infty}\left(c_{j j}^{(q)}\right)^{2} \sigma_{j j}}{\operatorname{Var}\left(y_{i t}\right)}, \quad i, j=1,2, \cdots, k
$$

相对方差贡献率 (RVC) 是根据第 $\mathrm{j}$ 个变量基于冲击 的方差对 $\mathrm{y}_{\mathrm{i}}$ 的方差的相对贡献度来观测第 $\mathrm{j}$ 个变量对 第 $\mathrm{i}$ 个变量的影响。如果模型满足平稳性条件, 则 $\mathrm{c}_{\mathrm{jj}}^{(\mathrm{q})}$ 随着 $\mathrm{q}$ 的增大呈几何级数性衰减, 所以只需取有限项 [12]。

\section{3.数据来源与数据处理}

本文主要选取 2014 年 8 月 1 日至 2015 年 4 月 30 日, 共计 639 天的单日猪肉、大豆、玉米价格数据, 其变 量分别用 PP 表示猪肉价格 (pork price), SP 表示大 豆价格 (soybean price), CP 表示玉米价格 (corn price)。 PP、SP、CP 的数据均来自贵州农经网。为消除时间
值, 因此它们均是平稳序列。但猪肉价格序列的原始 序列不为平稳序列, 经过一阶差分后, 变为平稳序列, 因此猪肉价格服从一阶单整, 可进行协整分析。

\subsection{Johansen 协整检验}

本文运用 Johansen 检验的方法来对模型进行协整检 验, 依据 $\mathrm{AIC}$ 和 SC 准则确定 VAR 模型的滞后阶数 为 6 阶。根据图 1 所示, 所有特征根的位置都处于单 位圆内, 因此表明所建立的模型为稳定的 VAR 模型。

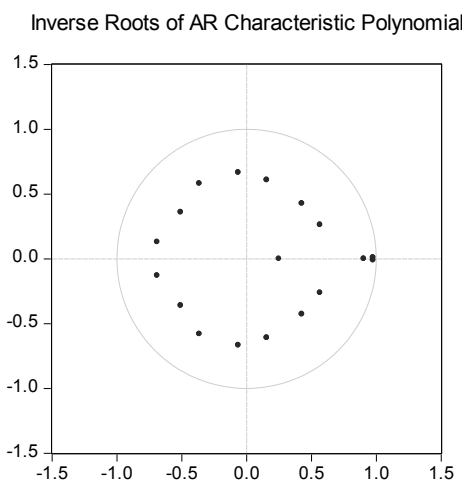

图 1. 特征根图形

\subsection{Granger 因果关系检验}

为了检验各序列之间是否存在因果关系, 通常对其进 
Risk Analysis and Crisis Response in Big Data Era (RAC-16)

Granger 因果关系检验。Granger 因果关系检验这一方 法解释了 $\mathrm{x}$ 是否引起 $\mathrm{y}$ 的问题, 主要看现在的 $\mathrm{y}$ 能够 在多大程度上被过去的 $\mathrm{x}$ 解释, 加入 $\mathrm{x}$ 的滞后值是否 使解释程度提高。 $5 \%$ 显著水平下的 Granger 因果关 系检验结果如表 2 所示。

表 2. $5 \%$ 的显著水平下 Granger 因果关系检验

Null Hypothesis（原假设）： F-Statistic Prob. 结论 LNSP does not Granger Cause LNCP $2.6213 \quad 0.0162$ 拒绝 LNCP does not Granger Cause LNSP $3.4838 \quad 0.0021$ 拒绝

LNPP does not Granger Cause LNCP $1.1389 \quad 0.3380$ 接受 LNCP does not Granger Cause LNPP 3.2760 0.0035拒绝

LNPP does not Granger Cause LNSP $2.4426 \quad 0.0242$ 拒绝 LNSP does not Granger Cause LNPP $2.2084 \quad 0.0407$ 拒绝 从表 2 的检验结果可知, 在 $5 \%$ 的显著水平下, 大豆价格 (LNSP) 与玉米价格 (LNCP) 互为格兰杰 原因, 猪肉价格 (LNPP) 与大豆价格 (LNSP) 互为 格兰杰原因, 玉米价格 (LNCP) 是猪肉价格 (LNPP) 的格兰杰原因, 但猪肉价格 (LNPP) 却不是玉米价 格（LNCP）的格兰杰原因。

\section{4 建立 VAR 模型}

LNPP $=0.060033+0.872502 *$

$\operatorname{LNPP}(-1)+0.052176 * \operatorname{LNPP}(-2)+0.040259 *$ $\operatorname{LNPP}(-3)+0.115794 * \operatorname{LNPP}(-4)-0.098492 *$ $\operatorname{LNPP}(-5)+0.000105 * \operatorname{LNPP}(-6)-0.005140 *$ $\operatorname{LNCP}(-1)-0.002138 * \operatorname{LNCP}(-2)-0.005420 *$ $\operatorname{LNCP}(-3)+0.008485 * \operatorname{LNCP}(-4)-0.011751 *$ $\operatorname{LNCP}(-5)+0.027958 * \operatorname{LNCP}(-6)-0.017931 *$ $\operatorname{LNSP}(-1)-0.012153 * \operatorname{LNSP}(-2)-0.018817 *$ $\operatorname{LNSP}(-3)+0.037502 * \operatorname{LNSP}(-4)+0.006479 *$ $\operatorname{LNSP}(-5)-0.004339 * \operatorname{LNSP}(-6)$

$\mathrm{R}^{2}=0.964687 \quad \mathrm{~F}=931.8459 \quad \mathrm{AIC}=-5.432120$ $\mathrm{SC}=-5.298535$

根据 AIC、SC 准则, 确定 VAR 模型的最大滞后阶数 为 6 。这为脉冲响应函数分析与方差分解提供了依据。

\section{5.贵州省猪肉价格、大豆价格与玉米价格的脉冲响应 函数与方差分解}

\section{1 脉冲响应函数分析}

脉冲响应函数是基于 VAR 模型的基础上建立的, 通过函数图像反应一个变量对另一个变量的传递 效用时滞与强度的动态变化。
由图 2 可知, 玉米价格 (LNCP) 对猪肉价格 (LNPP) 的冲击在期初为 0 , 后形成负向影响, 且 冲击逐渐增强, 在第 4 期时达到-0.002778 后, 冲击 小幅减弱, 第 5 期继续增强, 并在第 6 期达到负向最 大值 -0.003525 , 随后, 负向冲击逐渐减弱, 到第 10 期时冲击趋于零。大豆价格 (LNSP) 对猪肉价格 (LNPP) 的冲击在期初为零, 后开始形成负向冲击, 并逐渐增大, 在第 4 期时达到最大值- 0.003926 , 随后 趋于平稳, 维持在- 0.0032 至- 0.0035 的水平直至第 10 期。由此可以看出, 玉米价格与大豆价格对猪肉的价 格均为负向冲击, 但玉米价格对猪肉价格的冲击相对 较小, 大豆价格对猪肉价格的冲击较为强烈。

Response to Cholesky One S.D. Innovations ?2 S.E. Response of LNPP to LNCP

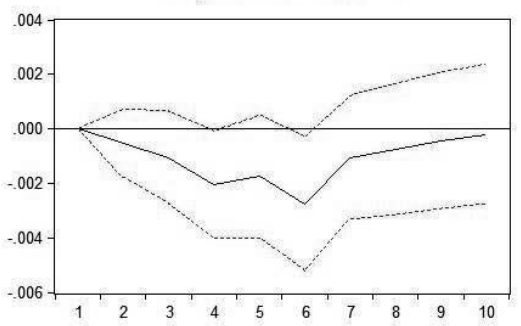

Response of LNPP to LNSP

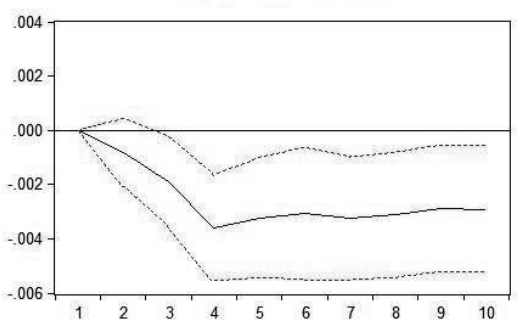

图 2. 玉米价格与大豆价格对猪肉价格的脉冲响应函数

由图 3 可知, 玉米价格 (LNCP) 对大豆价格 (LNSP) 的冲击在期初为正向, 后略微减弱, 在第 2 期降至 0.006383 后冲击又轻度加强, 在第 3 期达到 0.001044 后冲击逐渐减弱, 第 4 期降至 0.000557 后 冲击趋于平稳, 第 5 期时冲击又逐渐增强并持续至期 末。猪肉价格 (LNPP) 对大豆价格 (LNSP) 的冲击 在期初为负向, 在第 2 期达到负向最大值 -0.001970 后逐渐减弱, 在第 3 期时趋近于 0 , 直至第 5 期, 冲 击变为正向并逐渐增强,在第 6 期达到正向冲击最大 
Risk Analysis and Crisis Response in Big Data Era (RAC-16)

值 0.006035 , 随后冲击减弱并趋于平稳, 维持在 0.0048 左右的水平直至期末。由此看出, 猪肉价格对 大豆价格的冲击在期初为负向, 后变为正向, 玉米价 格对大豆价格的冲击一直为正向, 且玉米价格对大豆 价格的冲击较强。

Response to Cholesky One S.D. Innovations ?2 S.E.
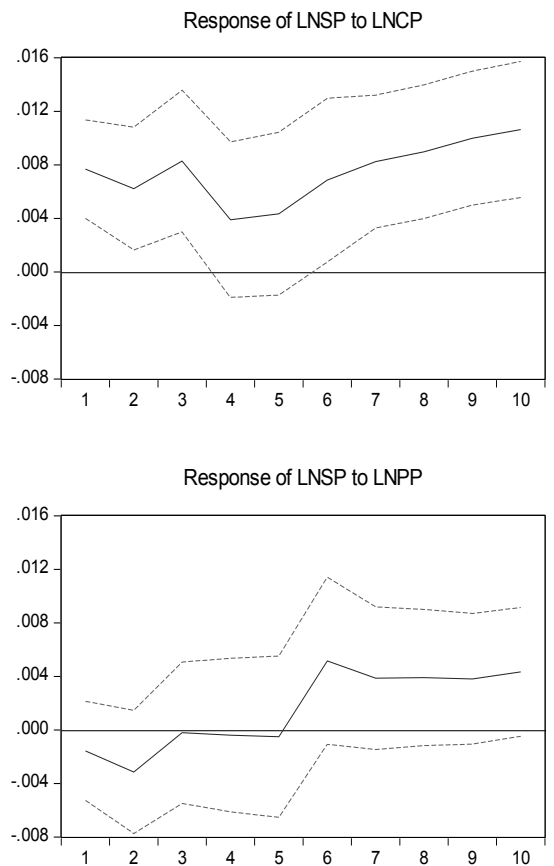

图 3. 玉米价格与猪肉价格对大豆价格的脉冲响应函数图

由图 4 可知, 猪肉价格 (LNPP) 对玉米价格 （LNCP）的影响为负向，在第 2 期达到负向最大值 -0.001982 后开始逐渐减弱并趋近于零, 直至第 5 期 开始转变为正向冲击且逐渐增强至第 6 期达到正向 最大值 0.005364 , 随后又开始逐渐减弱, 在第 8 期时 冲击开始趋近于零直至期末。大豆价格 (LNSP) 对 玉米价格 ( $\mathrm{LNCP})$ 的冲击在期初为零, 后开始变为 正向冲击并逐渐增强，在第 2 期时达到 0.008572 , 后 基本维持稳定直至第 5 期达到正向最大值 0.009648 , 随后开始减弱至第 6 期降至 0.001287 , 随后冲击开始 增强至第 7 期达到 0.006263 后趋于平稳直至期末。 由此看出, 猪肉价格对玉米价格的冲击一开始为负向 后转为正向但冲击并没有显著的影响, 大豆价格对玉 米价格的冲击一直为正向冲击, 中间虽然有减弱但总
体趋于稳定，且冲击强于猪肉价格。

Response to Cholesky One S.D. Innovations ?2 S.E. Response of LNCP to LNPP

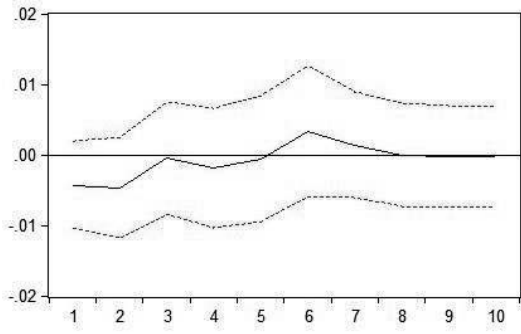

Response of LNCP to LNSP

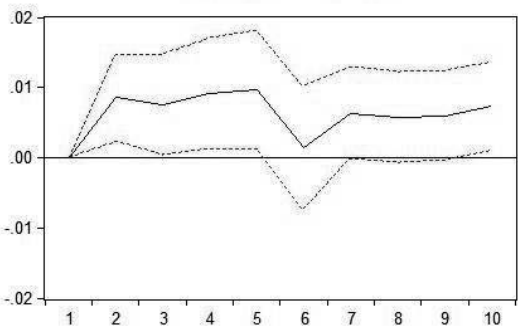

图 4. 猪肉价格与大豆价格对玉米价格的脉冲响应函数图

5.2 方差分解

方差分解结果见表 3、表 4、表 5。

表 3. 猪肉价格 (LNPP) 的方差分解结果

\begin{tabular}{rcccc} 
期间 & S.E. & LNPP & LNCP & LNSP \\
\hline 1 & 0.015768 & 100.0000 & 0.000000 & 0.000000 \\
2 & 0.020982 & 99.78078 & 0.065412 & 0.153803 \\
3 & 0.024770 & 99.05765 & 0.233751 & 0.708602 \\
4 & 0.028185 & 97.09087 & 0.717014 & 2.192121 \\
5 & 0.031784 & 96.37992 & 0.869243 & 2.750832 \\
6 & 0.034802 & 95.56684 & 1.358315 & 3.074845 \\
7 & 0.037327 & 95.30518 & 1.262603 & 3.432220 \\
8 & 0.039527 & 95.15046 & 1.162685 & 3.686859 \\
9 & 0.041516 & 95.10900 & 1.066361 & 3.824635 \\
10 & 0.043345 & 95.06026 & 0.980646 & 3.959090 \\
\hline
\end{tabular}


Risk Analysis and Crisis Response in Big Data Era (RAC-16)

表 4. 大豆价格（LNSP）的方差分解结果

\begin{tabular}{|c|c|c|c|c|}
\hline 期间 & S.E. & LNPP & LNCP & LNSP \\
\hline 1 & 0.015768 & 0.113207 & 2.714917 & 97.17188 \\
\hline 2 & 0.020982 & 0.371722 & 2.934739 & 96.69354 \\
\hline 3 & 0.024770 & 0.280273 & 3.753315 & 95.96641 \\
\hline 4 & 0.028185 & 0.236248 & 3.410947 & 96.35281 \\
\hline 5 & 0.031784 & 0.215249 & 3.361553 & 96.42320 \\
\hline 6 & 0.034802 & 0.612736 & 3.838797 & 95.54847 \\
\hline 7 & 0.037327 & 0.783348 & 4.540032 & 94.67662 \\
\hline 8 & 0.039527 & 0.943202 & 5.359384 & 93.69741 \\
\hline 9 & 0.041516 & 1.077170 & 6.340085 & 92.58274 \\
\hline 10 & 0.043345 & 1.249059 & 7.383079 & 91.36786 \\
\hline
\end{tabular}

表 5. 玉米价格 (LNCP) 的方差分解结果

\begin{tabular}{ccccc}
\hline Period & S.E. & LNPP & LNCP & LNSP \\
\hline 1 & 0.015768 & 0.313942 & 99.68606 & 0.000000 \\
2 & 0.020982 & 0.505506 & 98.60627 & 0.888226 \\
3 & 0.024770 & 0.415392 & 98.30362 & 1.280988 \\
4 & 0.028185 & 0.388125 & 97.79824 & 1.813634 \\
5 & 0.031784 & 0.350758 & 97.30462 & 2.344618 \\
6 & 0.034802 & 0.397103 & 97.45510 & 2.147798 \\
7 & 0.037327 & 0.379707 & 97.36185 & 2.258442 \\
8 & 0.039527 & 0.358373 & 97.30752 & 2.334111 \\
9 & 0.041516 & 0.340087 & 97.23994 & 2.419972 \\
10 & 0.043345 & 0.325222 & 97.06322 & 2.611562 \\
\hline
\end{tabular}

从表 3 中看出, 猪肉价格受自身价格影响最大, 占 $95 \%$; 其次受大豆价格的影响, 约占 $4 \%$; 受玉米 价格的影响最小, 约占 $1 \%$ 。从表 4 看出, 大大豆价 格受自身价格的影响最大, 约占 $91 \%$; 其次受玉米价 格的影响, 约占 $7.4 \%$; 受猪肉价格影响较小, 仅占 $1.2 \%$ 。从表 5 看出, 玉米价格受其自身价格的影响最 大, 约占 $97 \%$; 其次受大豆价格的影响, 约占 $2.6 \%$, 而猪肉价格对其的影响较小, 约占 $0.33 \%$ 。可见, 猪
肉价格受其自身的影响最大, 但其对玉米价格和大豆 价格的影响相对较小,玉米价格和大豆价格之间有一 定的互相影响作用, 但影响程度均较小。

\section{6.结论与建议}

本文运用 VAR 模型,对 2014 年 8 月 1 日至 2016 年 4 月 30 日的猪肉、大豆与玉米日价格的互动关系 研究。结果表明, 影响猪肉价格波动的主要因素是其 自身价格波动, 大豆价格与玉米价格对其有一定的影 响作用, 并且大豆价格对猪肉价格的影响程度比玉米 价格的影响更大。大豆价格和玉米价格会相互影响, 猪肉价格对大豆价格和玉米价格也会产生一定的影 响, 但对玉米价格的影响较小。

因此, 为了保障贵州省的猪肉价格稳定, 首先, 应建立猪饲料生产与监管体系, 保障玉米、大豆等产 品的价格稳定, 防止玉米、大豆价格出现剧烈波动而 影响猪肉价格稳定。其次, 应建立猪肉供需市场信息 平台, 定期更新猪肉及其相关数据信息, 确保市场信 息的公开性和流动性, 帮助养殖户和经销商做出合理 的生产经营决策, 从而促进猪肉价格的稳定。最后, 应加强建设生猪疾病防疫控制体系, 防止因猪瘟等重 大疫病的爆发对猪肉的供求产生剧烈影响, 从而影响 猪肉市场价格的稳定。

\section{Acknowledgements}

This study was support by Soft Science research project of Guizhou([2013]No.LKC2004), and humanities and social sciences research project of Guizhou education department (No.13GH032).

\section{致谢}

本研究得到了贵州省软科学研究项目 (黔科合体 $\mathrm{R}$ 字[2013]LKC2004 号) 和贵州省教育厅高校人文社会 科学研究项目 (13GH032) 的资助。

\section{参考文献}

[1] 国家统计局.2015 贵州统计年鉴.贵阳:中国统计 出版社,2015:47-48.

[2] 刘春. 贵州生猪标准化规模养殖现状及对策. 贵州 畜牧兽医,2012,35(3):39-40

[3] 朱满德,刘超,贾杰等. 贵州省具有发展生猪养殖 业的比较优势吗?一一基于成本收益数据的比较 分析.中国畜牧杂志,2013,49(6):14-18

[4] 站立强.生猪价格时间序列的规律性波形和成因 
Risk Analysis and Crisis Response in Big Data Era (RAC-16)

及调控.农业现代化研究,2014,35(1):25-28.

[5] 宁做凉,乔娟等.中国生猪价格波动的影响与成因 探究.中国畜牧经济.2010.46(2):52-56.

[6] 张立中,刘倩倩,辛国昌等.我国生猪价格波动调 控对策研究.经济问题探索.2013(11):117-122.

[7] 潘方卉,李翠霞等.我国生猪价格非线性波动规律 的实证研究.经济理论与实践,2014(2):20-25.

[8] 李威夷,生猪价格波动规律研究.中国农业科 学 院,2011:14-33.

[9] 张晓东,王超群,王宁等.基于 VAR 模型的生猪价 格波动的实证研究.安徽农业科学.2014,42(8)

[10] 康艺之,郑业鲁,林伟君等.我国猪市价格异常波
动预警及其对策研究.南方农村.2016(1):46-49.

[11] 赵瑞芗,陈会英,杨学成等.生猪价格风险预警模 型的建立与应用.运筹与管理.2008(4):128-131.

[12] 高铁梅.计量经济分析方法与建模.北京:清华大 学出版社.2006:267-290.

[13] G.Y. You, S.S Cao, J. Feng, S. Yu, An empirical research on the relationship between property insurance premiums and macroeconomic variables based on ARDL model. Journal of Risk Analysis and Crisis Response. 2014, 4(3): 175-181. 